\title{
FACILITATING INTERACTION WITH STEREOSCOPIC 3D DISPLAY DEVICES
}

\author{
Haiyue Yuan, Janko Ćalić \\ I-Lab, Centre for Vision, \\ Speech and Signal Processing \\ University of Surrey, UK \\ h.yuan@surrey.ac.uk; j.calic@ surrey.ac.uk
}

\author{
Ahmet Kondoz \\ Institute for Digital Technologies \\ Loughborough University \\ in London (LUiL), UK \\ a.kondoz@lboro.ac.uk
}

\begin{abstract}
In spite of a large body of research focused on 3D video technology, very little attention has been dedicated to the design practices of stereoscopic 3D video interaction devices. Interaction tasks such as pointing and selection are critical to the consumer's experience of the $3 \mathrm{D}$ video technology. This paper presents implementation and investigation of pointing modalities in the context of stereoscopic 3D display devices. The conducted investigation implements three pointing modalities: standard mouse-based interaction, virtual laser pointer implemented using Wiimote, and hand movement modality using Kinect. The study explores it's utilisation in interactive tasks such as two-handed interaction and zoom functionalities. Finally, we investigate the definition of interaction space for hand movement modality to facilitate effective and comfortable pointing.
\end{abstract}

Index Terms - Stereoscopic 3D video, Wiimote, Kinect, Interaction modality, Pointing, Fitt's Law, ISO 9241-9, Interaction space

\section{INTRODUCTION}

A considerable amount of research has looked into the development of intuitive interaction for using state-of-the-art consumer electronic devices such as Microsoft Kinect and Nintendo Wiimote. The consumer device manufacturers have mainly focused on the interaction for smart TV. The smart TV produced by Samsung captures the hand motion and gesture to offer smart interaction. The LG Magic Remote from LG smart TV enables users to point and click on the smart content, offering similar functionalities to the computer mouse. However, very little research has been addressing stereoscopic 3D video interaction in terms of interaction modality, user performance, user experience and user satisfaction. Unlike the 3D computer generated (CG) content, stereoscopic 3D video only provides pixel-based information of the left and right view in order to induce the illusion of depth. Therefore, the traditional 3D interaction techniques can not be applied to stereoscopic 3D video content due to nonexistent information of the scene geometry. A few researchers have addressed the intuitive interaction with video content, such as direct object manipulation video player $[1,2]$, but their focus has been solely on $2 \mathrm{D}$ video content. Compared to the $2 \mathrm{D}$ video content, stereoscopic $3 \mathrm{D}$ video provides an additional viewing dimension and thus offers more immersive experience to the audiences.

This paper presents a study of pointing modalities for interacting with stereoscopic $3 \mathrm{D}$ video content. The implementation of pointing modalities are developed based on different input de- vices, and the investigation is based on the multi-directional tapping task, as described in the ISO 9241-9 standard [3]. The Fitt's Law [4] model is used to analyse and compare the user performance. Pre- and post-experiment questionnaires are used to assess the subjective feedback of the devices. Furthermore, the challenge of the interaction space using hand movement modality based on Kinect is studied. The rest of this paper is organised as follows: Section 2 introduces the Fitts Law and ISO 9241-9 standard, Section 3 describes the process of the implementation and investigation, while the experiment results are presented in Section 4, finalising with the conclusions in Section 5.

\section{FITTS LAW AND ISO 9241-9 STANDARD}

Fitts' law is a model of human psychomotor behaviour based on a fundamental theorem of communication systems from Shannon's Theorem [5]. The realisation of movement in Fitts' law model is analogous to the transmission of information [6]. The movement task carried out in the human motor system over a certain period of time is similar to the bits of information transmitted over time (bits per second) in a communication system. The movement time (MT) is related with the effective movement distance (De) and effective target width (We).

$$
M T=a+b * \log _{2}\left(D_{e} / W_{e}+1\right)
$$

where $\mathrm{a}$ and $\mathrm{b}$ in Eq. 1 are constants empirically determined through the experiments. The log term is referred as effective index of difficulty (IDe) in Eq. 2, the unit for IDe is bits. The formulation for IDe is derived from Shannon formulation Eq. 2.

$$
I D_{e}=\log _{2}\left(D_{e} / W_{e}+1\right)
$$

The dependent measure of Fitts' law is throughput (TP). TP is defined in the Eq. 3 and carries the units of 'bits per second'.

$$
T P=I D_{e} / M T
$$

$W_{e}$ is derived from the observed distribution of selection coordinates in participants' trials as described in Eq. 4.

$$
W_{e}=4.133 * S D
$$

where SD is the standard deviation of the end-point positions. It is a convention to use a sub-range of end-point positions, which corresponds to around $96 \%$ of the distribution as the effective width. This range approximately equivalent to 4.133 standard deviations of the end positions [7].

The ISO 9241 standard is adopted in this study. It presents the requirements for ergonomic design of visual display terminals used 
Table 1. Questions from the post-experiment questionnaire.

\begin{tabular}{|l|l|}
\hline No. & Question \\
\hline 1 & The force required for actuation \\
2 & Smoothness during operation \\
3 & The mental effort required for operation \\
4 & The physical effort required for operation \\
5 & Accurate pointing was easy/difficult \\
6 & Operation speed was too fast/too slow \\
7 & Finger fatigue \\
8 & Wrist fatigue \\
9 & Arm fatigue \\
10 & Shoulder fatigue \\
11 & Neck fatigue \\
12 & General comfort \\
13 & Overall, the input device was easy to use \\
\hline
\end{tabular}

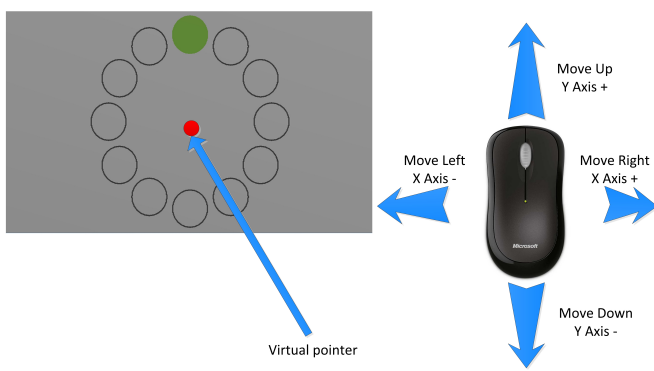

Figure 1. Implementation of mouse modality

for office work. Part 9 of this standardisation describes the requirements for non-keyboard input devices. The multi-directional tapping task in Annex B of Part 9 is used in this study. The primary dependent measure for ISO 9241-9 standard is throughput (TP). In addition, the post-experiment qualitative evaluation of the pointing devices is included in the ISO9241-9 to have participants subjectively assess the aspects of operation, fatigue, comfort, and overall usability. Post-experiment questionnaire is depicted in Table 1 .

\section{IMPLEMENTATION AND INVESTIGATION}

In this part, the implementation of interaction modality is presented, followed by introduction of the investigation in terms of experimental setup, design, and procedure.

\subsection{Interaction modality implementation}

Three modalities are implemented based on three different input devices, using the Wiiyourself library and Windows Kinect SDK.

Mouse modality: The main aim of introducing the mouse modality was to benchmark virtual laser pointer and hand movement modalities. The mouse modality was implemented by using the traditional mouse. The user manipulates the mouse to control the movement of the virtual pointer on the screen to complete the pointing task. Left click of the mouse indicates the confirmation of selection (see Figure 1).

Virtual laser pointer modality: As the remote controller is the dominant pointing device used to interact with the TV set. Wiimote is utilised to mimic and model the experience of using the remote controller, adding additional functionalities to achieve the required task that needs for this experiment. The virtual laser pointer modality is implemented using a Wiimote with MotionPlus, and a Kinect. The Kinect was placed at the central position under the bottom of the display, and the Wiimote was hand held

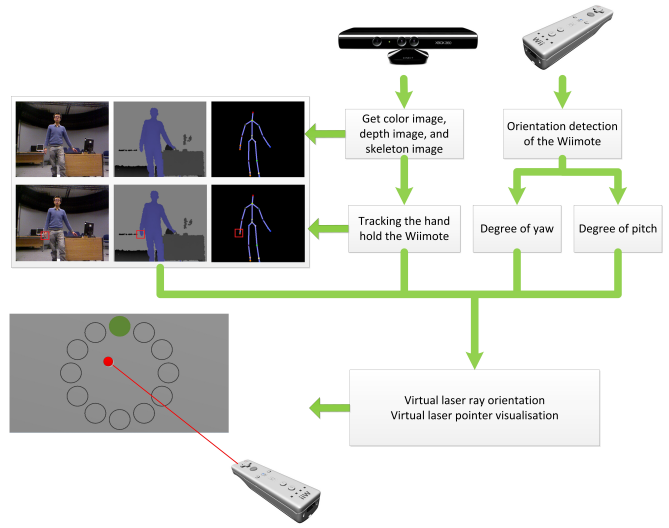

Figure 2. Implementation of virtual laser pointer modality

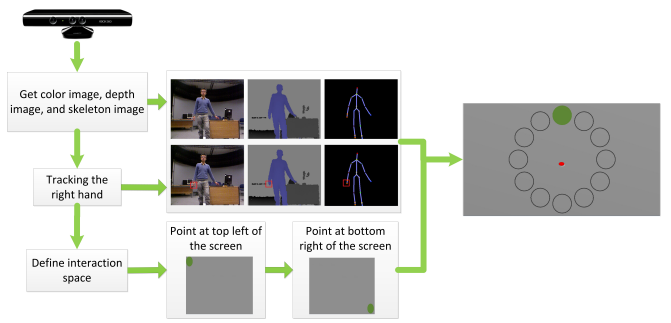

Figure 3. Implementation of hand modality

by the participant. The Kinect tracked the right hand of the participant. The 3D coordinates of the tracked right hand located the source of the virtual laser ray. Wiimote with MotionPlus was used to detect the degree of pitch and yaw of Wiimote, which can indicate the orientation of the virtual laser ray (see Figure 2). The combination of the hand coordinates and orientation enables user to hold the Wiimote as a virtual laser pointer to move the pointer on screen to complete the pointing task.

Hand movement modality: This modality is implemented using the Kinect only. The Kinect is placed at the center position on top of the display. For hand movement modality, the participants control the pointer on the screen by moving their right hands. Each participant needed to define an interaction space before doing the experiment. To define the interaction space is to map the physical movement to the virtual movement on the screen. The aim is to find out preferred interaction space that can offer effective interaction. There were two steps to define the interaction space. Firstly, the participant was instructed to move the right hand to a position where they can comfortably move the pointer to the top left corner of the screen. Next step was to ask the participant to do the same action but towards the right bottom corner of the screen. The coordinates of each position to form a virtual rectangle interaction space in front of the participant are recorded. The participants have repetitive chances to define and test the interaction space in order to achieve comfortable pointing (see Figure 3).

\subsection{Participants and apparatus}

Fifteen subjects were recruited to participate in the study as volunteers. They were aged from 22 to 30 . All participants were right handed. Each participant needs to complete a pre-experiment questionnaire before conducting the experiment. All of them have previous experience of watching 3D movies, and previous experience of playing 3D games using computer or game console. In addition, each participant took a Randot stereo acuity test, and all 
of them had accepted stereo perception.

The experiment took place in a laboratory equipped with a 46" JVC stereoscopic display with passive polarisation glasses (Model number GD-463D10). The resolution of the display was 1920x1080 with the recommended viewing distance of 2 meters from the screen.

\subsection{Procedure \& Design}

The pointing task was designed based on the ISO 9241-9 multidirectional tapping task. The participants were presented with 12 circular targets, arranged in a circle in the centre of the screen. Each target is separated by 30 degrees. Participants were instructed to point each highlighted target as soon as possible using the input device.

This study used a $6 \times 5 \times 3$ within-subjects design. The independent variables were 6 conditions of different targets size and distance, 5 disparity levels, and 3 pointing modalities. The dependent variables were movement time (s), and throughput (bits per second). In addition, 5 disparity levels were utilised: -20 pixel, -10 pixels, 0 pixels, 10 pixels, and 20 pixels ( 1 pixel approximately equals $0.05 \mathrm{~mm}$ on the screen). All targets were presented at a consistent depth in each condition, so that the participants had the same visual depth for all targets in each condition. However, the depth of the targets was varied between each conditions. In summary, there were $30(6 \times 5)$ scenarios for each pointing modality. Each of the 15 participants completed 12 trials ( 1 target is 1 trial) over 30 scenarios for each pointing modality. There were three different modalities in total. Therefore there were $3 \times 6 \times 5 \times 12 \times 15=16200$ trials in total. 15 participants were divided into three groups. The order of the presentation of the pointing tasks to each group was counterbalanced using a $3 \times 3$ Latin square to offset any learning effects. The dependent variables in this experiment were movement time (MT) and throughput (TP).

\section{EXPERIMENT RESULTS}

\subsection{Fitting of Fitts's law model}

The data is gathered from all participants and took the average values for each condition. There were 30 conditions for each pointing modality. According to the Eq. 1, and Eq. 2, the following Eq. 5 are obtained as the Fitts' law model.

$$
M T=a+b * I D_{e}
$$

The IDe is determined by the effective distance De and effective target width We. As the circular arrangement for the targets is used in this study, the effective width and effective distance need to be calculated according to the approach angle of pointing.

The least-squares linear regression is used to find the intercept (a) and slope (b) parameters of the Fitts' law Eq. 5. The following Eq. 6, Eq. 7, and Eq. 8 represent the Fitts' law equation for the mouse, virtual laser pointer, and hand movement modality respectively.

$$
\begin{aligned}
& M T=0.2482+0.1998 * I D_{e}, r^{2}=0.8288 \\
& M T=0.3796+0.2114 * I D_{e}, r^{2}=0.9026 \\
& M T=0.4995+0.2854 * I D_{e}, r^{2}=0.8884
\end{aligned}
$$

The $\mathrm{r}^{2}$ of the linear regression between MT and IDe for all pointing modalities approximately equals to 0.9 , which indicates a good fit to the Fitts law model. As stated in [7], the intercept should be less than 0.4 seconds to prove the legitimacy of the experimental
Table 2. Statistical report. (Significant effects are marked $*$ for $\mathrm{p}<0.05$, $* *$ for $\mathrm{p}<0.01$ and $* * *$ for $\mathrm{p}<0.001$ )

\begin{tabular}{|l|l|l|}
\hline Factor & Movement time & Throughput \\
\hline & $\mathrm{F}(\mathrm{p})$ & $\mathrm{F}(\mathrm{p})$ \\
\hline (P)ointing modality & $577.34(* * *)$ & $497.68(* * *)$ \\
\hline (C)ondition & $284.29(* * *)$ & $60.43(* * *)$ \\
\hline (D)isparity level & $5.48(*)$ & $0.62(0.65)$ \\
\hline P x C & $12.62(* * *)$ & $1.77(0.09)$ \\
\hline P x D & $3.14(*)$ & $1.75(0.11)$ \\
\hline C x D & $1.81(0.06)$ & $1.54(0.12)$ \\
\hline
\end{tabular}

Table 3. Comparison of overall throughput
\begin{tabular}{|l|l|}
\hline Modality & Throughput mean(sd) \\
\hline Mouse & $3.44(0.25)$ \\
\hline Virtual Laser Pointer & $3.25(0.14)$ \\
\hline Hand Movement & $1.96(0.35)$ \\
\hline
\end{tabular}

design. The value of the intercept for hand movement modality is 0.4995 . The cause of the large slope is the dwell time due to the hand fatigue during the experiment. The analysis of the postexperiment questionnaire also proves the hand and arm fatigue problem in case of hand movement modality.

\subsection{Effect of different factors}

Results were analysed using ANOVA and Tukey-Kramer multiple comparisons at the 5\% significance level. The statistical report is presented in Table 2.

Overall, there was a significant main effect of both pointing modality and condition on the MT and TP.The comparison of MT between modalities for different conditions suggests that participants spent less time using both mouse and Wiimote to complete the task compare with using Kinect for each condition. The comparison between mouse and virtual laser pointer modality suggest that on average participant can achieve quicker pointing action using virtual laser pointer. Furthermore, the post-hoc Tukey-Kramer test was applied to compare the correlation of MT between pointing modalities. The results suggest that there is significant difference of MT between different pointing modalities. As well as the pointing modality, the Tukey-Kramer test was employed to compare MT between conditions. The results confirm that there is a significant effect of condition to the MT. In addition, the analysis revealed a significant effect of pointing modalities to the TP.

The experiments demonstrated that the virtual laser pointer and mouse has similar TP, which was always greater than hand movement modality. The larger the value of TP represents the better assessment of pointing modalities. The mean and standard deviation of the overall TP are shown in Table 3. The post-hoc Tukey-Kramer test of TP between pointing modalities proves the significance of pointing modalities towards the TP.

The impact of the disparity level on the MT and TP is also investigated. Refer to the Table 2, the results revealed that there is a impact of disparity level on MT at 5\% significance level, however there is no significant effect to TP.

Furthermore the interaction effect between different factors to the MT and TP suggest that cross all conditions, the participants spent more time to complete the task using Kinect, while there is no significant difference between the other two modalities. The comparison between disparity levels can not reveal significant difference. In regard to the investigation of TP, the results suggest that the mouse and virtual laser pointer can offer similar TPs across all conditions. The hand movement modality provides the worst TP for all conditions. 
The difference of MT and TP between virtual laser pointer and hand movement is due to the movement required in the experiment. For virtual laser pointer, the participants had minimum arm movement and shoulder movement. Instead, only wrist rotation and little hand movement were needed from the participants to complete the pointing task. However, participants need to have relative larger movement of hand and shoulder using Kinect in order to manipulate the pointer. The subsequent arm and shoulder fatigue can influence the user performance. From this point of view, virtual laser pointer modality using Wii can provide more efficient pointing than hand movement modality using Kinect.

\subsection{Interaction space}

Each participant needed to determine an interaction space before starting the pointing task using hand movement modality. The aim is to understand and investigate how the participants define the interaction space to facilitate the effective and comfortable interaction.

The hand movement modality proposed in this study involves only two degree-of-freedoms (DoFs). One is the movement along $\mathrm{x}$-axis, which corresponds left and right movement of the hand. Another one is the movement along y-axis, which corresponds up and down movement of the hand. Due to the characteristic of the stereoscopic 3D video content, the user just need to select the object at the left image, the disparity information can find the corresponding object at the right image. Therefore only two DoFs are enough to choose any objects at any level of depth for stereoscopic $3 \mathrm{D}$ video content.

The real 3D coordinates of the right hand and the coordinates of the right hand in the skeleton image for each participant were recorded during the experiment. According to the data, the interaction space can be categorised into three groups. Based on coordinates of the skeleton image (resolution of 640 x 480) captured by the Kinect, the average size of small interaction space approximately equals to 7200 square pixels (90 pixels $\times 80$ pixels); the medium interaction space is about 16500 square pixels (150 pixels $\times 110$ pixels); the size of large interaction space is around 37400 square pixels ( 220 pixels $\times 170$ pixels). In addition, the mean and standard deviation of MT and TP for different sizes of the interaction spaces are shown in Table 4.

Table 4. MT and TP for different interaction spaces

\begin{tabular}{|l|l|l|}
\hline Interaction Spaces & MT(s) & TP (bits/s) \\
\hline Small & $1.34(0.02)$ & $1.8(0.05)$ \\
\hline Medium & $1.11(0.08)$ & $2.14(0.17)$ \\
\hline Large & $1.07(0.1)$ & $2.13(0.28)$ \\
\hline
\end{tabular}

The results revealed that the participants spent more time to complete the pointing task with smaller interaction space. The size of the interaction space is inversely proportion to the movement of hand required in the real world. Therefore the larger movement of the pointer on the screen requires smaller movement of the hand. This could result the difficulty to easily move the pointer to the target position with rather smaller hand movement. Therefore it might require more time to accurately point at the target with smaller interaction space. However, the difference between different size of the interaction space is not large. In regards to the analysis of TP, the results suggested that the usability of using medium and large interaction space is better than the usability of using small interaction space. In addition, we measured how far the hand away from the body while using hand movement modality for interaction spaces. For the small interaction space, the participants stretched their hands approximately 0.2 meters away from the body to do the experiment. The distance was around 0.3 meters and 0.4 meters for the medium and large interaction space respectively. This distance is proportional to the size of the interaction spaces. With longer distance needed to stretch the arm for the larger size of the interaction space, the more effort is required to complete interaction task.

\section{CONCLUSION}

In this paper, a study of pointing modalities for stereoscopic 3D TV using ISO 9241-9 standard multi-directional tapping task is presented. Three different pointing modalities are implemented using mouse, Wiimote, and Kinect respectively. The Fitts' law model and qualitative evaluation were used to compare the pointing modalities. The movement time and throughput for each pointing task in relation to different conditions is measured, and the main findings suggest that the target size, the target distance, and the pointing modality has significant impact on movement time and throughput. However the analysis of disparity level failed to find any significant effect. The experiment results suggest that the mouse and virtual laser pointer modalities tend to achieve better user performance than hand movement modality using Kinect. Furthermore, the investigation of hand movement modality looked into the issue of defining the individual interaction space. The analysis indicated that either too small or too big interaction space could result worse overall usability of input device.

\section{ACKNOWLEDGMENT}

This work was supported partially by the ROMEO project (grant number: 287896), which was funded by the EC FP7 ICT collaborative research programme

\section{REFERENCES}

[1] Thorsten Karrer, Malte Weiss, Eric Lee, and Jan Borchers, "Dragon: a direct manipulation interface for frame-accurate in-scene video navigation," in Proceedings of the twenty-sixth annual SIGCHI, 2008, CHI '08, pp. 247-250.

[2] Pierre Dragicevic, Gonzalo Ramos, Jacobo Bibliowitcz, Derek Nowrouzezahrai, Ravin Balakrishnan, and Karan Singh, "Video browsing by direct manipulation," in Proceedings of the twenty-sixth annual SIGCHI, 2008, CHI '08, pp. 237-246.

[3] "International Organization for Standardization, ISO 92419:2000, Ergonomic requiremnets for office work with visual dusplay terminals - Part 9: Requirements for non-keyboard input devices," 2000.

[4] Paul M Fitts, "The information capacity of the human motor system in controlling the amplitude of movement.," Journal of experimental psychology, vol. 47, no. 6, pp. 381, 1954.

[5] C. E. Shannon, The mathematical theory of communication, Urbana: University of Illinois Press, 1949.

[6] I. Scott MacKenzie, "Fitts' law as a research and design tool in human-computer interaction," Hum.-Comput. Interact., vol. 7, no. 1, pp. 91-139, Mar. 1992.

[7] R. William Soukoreff and I. Scott MacKenzie, “Towards a standard for pointing device evaluation, perspectives on 27 years of fitts' law research in hci," Int. J. Hum.-Comput. Stud., vol. 61, no. 6, pp. 751-789, Dec. 2004. 